\title{
ON THE EXISTENCE OF TOTALLY INHOMOGENEOUS SPACES ${ }^{1}$
}

\author{
BRIAN M. SCOTT
}

ABSTRACT. A property of total inhomogeneity for topological spaces is defined and is shown to be stronger than that of rigidity (i.e., of having trivial autohomeomorphism group). It is further shown that compact, rigid, Hausdorff spaces are totally inhomogeneous, and that totally inhomogeneous spaces exist in profusion as dense subspaces of a class of locally compact spaces.

De Groot has studied in [3] the existence of spaces having specified autohomeomorphism groups, and in particular the existence of rigid spaces, i.e., spaces admitting only the identity autohomeomorphism. In this note we investigate the existence of spaces satisfying the stronger property of total inhomogeneity and the relation of such spaces to rigid spaces.

Definition. A space $X$ is totally inhomogeneous iff given $x, y \in X$ with $x \neq y$, the spaces $X \backslash\{x\}$ and $X \backslash\{y\}$ are not homeomorphic.

Clearly, if $X$ is totally inhomogeneous, then $X$ is rigid. The following example shows that the converse implication fails, even for locally compact metric spaces.

Example. Let $G$ be the directed graph of Figure 1; the automorphism group of $G$ is isomorphic [2] to $Z_{3}$. Let $P_{4}$ be as in [3]. (We sketch the construction: Let $D$ be the unit disk, and let $a_{1}, a_{2}, \ldots$ be a countable dense subset of the interior of $D$. Let $V_{1}$ be the open $r_{1}$-ball about $a_{1}$, where $r_{1}$ is chosen so that $V, \subseteq D$, and let $F_{1}$ be a 5-bladed "propeller" contained in $V_{1}$ and centered at $a_{1}$. Given $r_{i}, V_{i}, F_{i}$ for $i<n$, let $a_{n}^{\prime}$ be the first term of the sequence $a_{1}, a_{2}, \ldots$ such that $a_{n}^{\prime} \notin \bigcup\left\{F_{i}: i<n\right\}$. Let $V_{n}$ be the open $r_{n}$-ball about $a_{n}^{\prime}$, where $r_{n}<r_{n-1} / 2$ is such that $V_{n} \subseteq$ $D \backslash \bigcup\left\{F_{i}: i<n\right\}$, and let $F_{n}$ be an $(n+4)$-bladed propeller contained in $V_{n}$

Received by the editors May 21, 1973 and, in revised form, April 3, 1974.

AMS (MOS) subject classifications (1970). Primary 54G15; Secondary 54B05, $54 \mathrm{E} 45$.

Key words and phrases. Rigid space, totally inhomgeneous space, locally compact space.

1 During the preparation of this paper the author was a National Science Foundation Graduate Fellow. 
and centered at $a_{n}^{\prime}$. The space $P_{4}$ is $D \backslash \bigcup\left\{\operatorname{Int}\left(F_{n}\right): n=1,2, \ldots\right\}$, and is compact, connected, and rigid.) $P_{4}$ can be oriented and given "endpoints" by choosing $p_{0}, p_{1} \in P_{4} \cap \operatorname{Bdry}(D)$ on a diameter of $D$. Now replace each edge of $G$ by a copy of $P_{4}$ in the appropriate orientation, and call the resulting space $Y$. The autohomeomorphism group of $Y$ is [3] also isomorphic to $Z_{3}$. Form $X \subseteq Y$ by deleting the center of the 5 -bladed propeller of the copy of $P_{4}$ replacing the edge $v_{0} v_{1}$ of $G$, and let $x, y$ be the centers of the 5-bladed propellers of the copies of $P_{4}$ replacing $v_{1} v_{2}$ and $v_{2} v_{0}$. It is not hard to see that $X$ is rigid, metric, and locally compact, but that $X \backslash\{x\}$ is homeomorphic to $X \backslash\{y\}$.

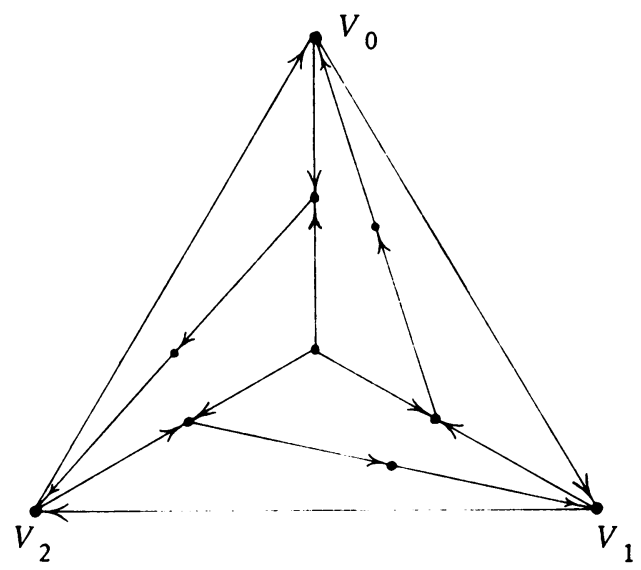

Figure 1

However, we do have

Theorem 1. If $X$ is a compact, rigid, Hausdorff space, then $X$ is totally inhomogeneous.

Proof. Suppose not; suppose $x \neq y$, but $h: X \backslash\{x\} \rightarrow X \backslash\{y\}$ is a homeomorphism. Let $\mathcal{F}=\{V \backslash\{x\}: x \in V$ and $V$ is open $\}$, and let $A=\bigcap\{c l(b(F))$ : $F \in \mathcal{F}\}$. $X$ is a compact and $h(\mathcal{F})$ is a filterbase on $X$, so $A \neq \varnothing$. Let $a \neq y$, say $a=b(z)$ for some $z \in X \backslash\{x\}$. Pick disjoint open neighborhoods $V$ and $W$ of $x$ and $z$, respectively, and let $F=V \backslash\{x\} \in \mathcal{F}$; then $b(W)$ is open, and $h(W) \cap h(F)=\varnothing$, so $a \notin A$, and $A=\{y\}$. In fact, if $V$ is any neighborhood of $y$, since $X \backslash V$ is compact, this argument shows that there are $F_{1} \ldots, F_{n}$ $\epsilon \mathcal{F}$ such that for $1 \leq i \leq n, \bigcap\left\{b\left(F_{i}\right)\right\} \subseteq V$; and $\bigcap\left\{h\left(F_{i}\right)\right\}=b\left(\bigcap\left\{F_{i}\right\}\right) \epsilon$ $h(\mathfrak{F})$, so $h(\mathcal{F})$ converges to $y$. Hence [1] $h$ extends to a $1-1$, continuous map of $X$ onto $X$ which, $X$ being compact Hausdorff, is a homeomorphism. 
Totally inhomogeneous spaces exist in profusion. Guaranteeing this is

Theorem 2. Let $\kappa$ be an infinite cardinal, and let $\lambda=2^{\kappa}$. Let $(X, \mathcal{U})$ be a compact Hausdorff space of cardinality $\lambda$ such that if $V \in \mathcal{U}$ and $V \neq \varnothing$, then $|V|=\lambda$, and suppose that $X$ has a basis of cardinality $\kappa$. Then there are subspaces $R_{a}, \alpha<\lambda$, each totally inhomogeneous, dense in $X$, and of cardinality $\lambda$, which are pairwise disjoint and pairwise nonhomeomorphic.

Proof. Call $A \subseteq X$ a $\kappa-G_{\delta}$ (resp. $\kappa-F_{\sigma}$ ) set iff $A$ is the intersection (resp. union) of $\kappa$ open (resp. closed) sets in $X$. Let $\mathcal{C}=\{f: f$ is continuous, $\operatorname{dom} f \subseteq X$, and $\operatorname{ran} f \subseteq X\}$. Say $f \in \mathcal{C}$ is a displacement iff for some $A \subseteq \operatorname{dom} f, f(A) \cap A=\varnothing$ and $|f(A)|=\lambda$, and let $\mathcal{C}_{0}=\{f \in \mathcal{C}: f$ is a displacement and $\operatorname{dom} f$ is a $\kappa \cdot G_{\delta}$ set $\}$. Since there are only $\lambda \kappa \cdot G_{\delta}$ subsets of $X$, each of which has a dense subset of cardinality $\kappa$, it follows that $\left|\mathcal{C}_{0}\right|=\lambda$. An immediate consequence of Theorem 2.2 of [4] is

Lemma 1. If $f \in \mathcal{C}$ is a displacement, then there is $g \in \mathcal{C}_{0}$ such that $f=g \uparrow \operatorname{dom} f$.

We are now in a position to construct the $R_{\alpha}$ 's.

Index $\mathcal{C}_{0}=\left\{f_{\alpha}: \alpha<\lambda\right\}, \mathcal{K}=\{K \subseteq X: K$ is compact and $|K|=\lambda\}=$ $\left\{K_{\alpha}: a<\lambda\right\}$. Let $T=\left\{t_{\alpha}: \alpha<\lambda\right\}$ be an enumeration of $\lambda \times \lambda \times \lambda$. Finally, let $\mathcal{U}=\left\{U_{a}: \alpha<\lambda\right\}$ enumerate $\mathcal{U}$.

If points, $p_{a}, q_{a}, k_{a} \in X$ have already been chosen for $\alpha<\gamma<\lambda$, suppose that $t_{y}=\langle\delta, \xi, \eta\rangle$, and choose $p_{y}, q_{y}, k_{y} \in X$ distinct from all $p_{a}$, $q_{a}, k_{a}$ for $\alpha<\gamma$ as follows: pick $p_{\gamma} \in \operatorname{dom} f_{\delta}$ such that $f_{\delta}\left(p_{\gamma}\right) \neq p_{y}$ and, if possible, such that $p_{\gamma} \in U_{\xi}$ (if every point of $U_{\xi} \cap\left\{x \in \operatorname{dom} f_{\delta}: f_{\delta}(x)\right.$ $\neq x\}$ has already been chosen, we drop the requirement that $p_{\gamma} \in U_{\xi}$ ); let $q_{y}=f_{\delta}\left(p_{y}\right)$, and pick $k_{\gamma} \in K_{\delta}$ distinct from $p_{\gamma}$ and $q_{y}$. This is always possible for $\gamma<\lambda$, since $f_{\delta} \in \mathcal{C}_{0}$ and $\left|K_{\delta}\right|=\lambda$.

Now, for $\alpha<\lambda$, let $R_{\alpha}=\left\{p_{y}: t_{y}=\langle\delta, \eta, \alpha\rangle\right.$ for some $\left.\delta, \eta<\lambda\right\} \cup$ $\left\{k_{y}: t_{y}=\langle\delta, \eta, a\rangle\right.$ for some $\left.\delta, \eta<\lambda\right\}$; clearly $\left|R_{a}\right|=\lambda$. Also, if $V \in \mathcal{U}$, then there is $W \in \mathcal{U}$ such that $\varnothing \neq W \subseteq \bar{W} \subseteq V$; and $\bar{W} \in \mathcal{K}$, so $\left|V \cap R_{a}\right| \geq$ $\left|\bar{W} \cap R_{a}\right|=\lambda$ for any $\alpha<\lambda$. It follows immediately that the $R_{a}$ are pairwise disjoint, dense subsets of $X$. To show that they are pairwise nonhomeomorphic and totally inhomogeneous, we actually prove more: if $V, W \in \mathcal{U}$ are such that $\varnothing \neq V \cap R_{\alpha} \neq W \cap R_{\beta} \neq \varnothing$, then $V \cap R_{\alpha}$ and $W \cap R_{\beta}$ are not homeomorphic. For if $h: V \cap R_{a} \rightarrow W \cap R_{\beta}$ is a homeomorphism, choose $x \epsilon$ $\left(V \cap R_{\alpha}\right) \backslash\left(W \cap R_{\beta}\right)$ [if $V \cap R_{\alpha} \subseteq W \cap R_{\beta}$, consider $b^{-1}$, and choose $x \in$ 
$\left.\left(W \cap R_{\beta}\right) \backslash\left(V \cap R_{a}\right)\right]$; then $h(x) \neq x$, so there are disjoint $U, G \dot{\epsilon} \mathcal{U}$ such that $x \in U \subseteq V, h(x) \in G \subseteq W$, and $h\left(U \cap R_{\alpha}\right)=G \cap R_{\beta}$. Note that $\left|G \cap R_{\beta}\right|$ $=\lambda$, so $h$ is a displacement, and for some $f_{y} \in \mathcal{C}_{0}, h=f_{y} \uparrow\left(V \cap R_{\alpha}\right)$. Also, for some $\delta<\lambda, U=U_{\delta}$. Let $\eta<\lambda$ be such that $t_{\eta}=\langle\gamma, \delta, \alpha\rangle$; then $\left|U \cap\left\{y \in \operatorname{dom} f_{y}: f_{y}(y) \neq y\right\}\right|=\lambda$, so $p_{\eta} \in R_{a} \cap U_{\delta} \subseteq R_{\alpha} \cap V$, and $h\left(p_{\eta}\right)=$ $f_{\gamma}\left(p_{\eta}\right)=q_{\eta} \notin R_{\beta}$, a contradiction.

Two corollaries to the proof of Theorem 2 are worth noting:

Corollary 1. Theorem 2 still holds if "compact Hausdorff space" is replaced by "locally compact Hausdorff space"; the proof can be carried out in the one-point compactification.

Corollary 2. There are $2^{\lambda}$ totally inhomogeneous subspaces of $X$, each of cardinality $\lambda$ and dense in $X$, which are pairwise nonhomeomorphic (but not pairwise disjoint).

Proof. At each stage of the construction choose six points, $p_{\alpha^{\prime}}^{i} q_{\alpha}^{i}, k_{\alpha}^{i}$, for $\alpha<\lambda$ and $i=0,1$, such that if $t_{\alpha}=\langle\gamma, \delta, \eta\rangle$, then $q_{\alpha}^{i}=f_{\gamma}\left(p_{\alpha}^{i}\right) \neq p_{\alpha}^{i}$, $k_{a}^{i} \in K_{\gamma}$, and, if possible, $p_{a}^{i} \in U_{\delta}$. (As before, all points are chosen to be distinct.) Then for each $\phi: \lambda \rightarrow\{0,1\}$, let $R_{\phi}=\left\{p_{a}^{\phi(\alpha)}\right\} \cup\left\{k_{a}^{\phi(a)}\right\}(a<\lambda)$.

Say a space has property $P$ if no two distinct, nonempty open subsets are homeomorphic: e.g., the $R_{a}$ 's constructed in the proof of Theorem 2 have property $P$. They are, however, very nonconstructive. Using far more constructive methods, Lozier [5] produces noncompact, 0-dimensional spaces $X^{\kappa}$ of any infinite cardinality $\kappa$, in which any two distinct points have different values under a topologically invariant, ordinal-valued function which is unchanged by restriction to open subspaces: consequently, $X^{K}$ has property $P$. It is, however, not the case that total inhomogeneity implies property $P$, as is easily seen from the following

Example. Let $X_{0}$ be a closed line segment in $E^{2}$ of length $l$, and let $x_{0}$ be its midpoint. Form $X_{1}$ by attaching a segment of length $1 / 2$ to $x_{0}$, and let $x_{1}, x_{2}$, and $x_{3}$ be the midpoints of the "arms" of $X_{1}$. Form $X_{2}$ by attaching two segments to $x_{1}$, three to $x_{2}$, and four to $x_{3}$ in such a way that the new "arms" have length $\leq 1 / 4$ and do not intersect $X_{1}$ except at $x_{1}, x_{2}$ " and $x_{3}$. Continue in this fashion, and let $X=\bigcup\left\{X_{n}: n \in \omega\right\}$. Let $Y$ be a copy of one of the components of $X \backslash\left\{x_{0}\right\}$, and let $Z$ be the disjoint union of $X$ and $Y$. Clearly $Z$ does not have property $P$, and it is not hard to see that $Z$ is totally inhomogeneous.

I wish to thank the referee for several valuable suggestions. 


\section{REFERENCES}

1. J. Dugundji, Topology, Allyn and Bacon, Boston, Mass., 1966. MR 33 \#1824.

2. R. Frucht, Graphs of degree three with a given abstract group, Canad. J. Math. 1 (1949), 365-378. MR 11, 377.

3. J. de Groot, Groups represented by homeomorphism groups. I, Math. Ann. 138 (1959), 80-102. MR 22 \#9959.

4. J. de Groot and R. H. McDowell, Autohomeomorphism groups of 0-dimensional spaces, Compositio Math. 15 (1963), 203-209. MR 27 \#4216.

5. F. W. Lozier, A class of compact rigid 0-dimensional spaces, Canad. J. Math. 21 (1969), 817-821. MR 39 \#6243.

DEPARTMENT OF MATHEMATICS, UNIVERSITY OF WISCONSIN, MADISON, WISCONSIN 53706

Current address: Department of Mathematics, Cleveland State University, Cleveland, Ohio 44115 